Military Technical College
Cairo, Egypt

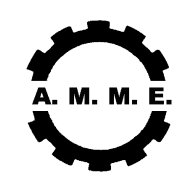

$12^{\text {th }}$ International Conference

on Applied Mechanics and

Mechanical Engineering (AMME)

\title{
A CRACK PATH-BASED MODEL TO ASSESS MICROSTRUCTURE INCLUSIONS RANDOMNESS
}

\author{
MOHAMED ${ }^{*}$ M.A.S.
}

\begin{abstract}
Discussed are the two main phenomena often observed in engineering materials prior to its fracture; namely dislocations-based strain hardening and the crack governing factors to steer its direction of propagation. The study investigates the micro displacements of these two mechanisms as monitored in a soft matrix full of hard obstacles (constraints).

Based on a marked similarity with an Artificial Intelligence method called Uphill Climbing, a cellular model is so constructed that relevant formulas of plane strain analysis are properly included. In the model, the magnitude and direction of each successive micro displacement are optimized with reference to a globally updated potential objective and a locally selected stressed spots.

It is found that for a randomly uniform obstacle distribution, low values of mean obstacle density lead to a direction-independent resistance against defects' micro displacements. As obstacle density increases, such a resistance shows a direction trend. Depending on the dislocation hardening history, the $100 \%$ full loading results in several independent segments of crack paths that may either finally meet or distantly lock apart, in which cases the fractured surfaces reflect relevant distinct zones.
\end{abstract}

\section{KEY WORDS}

Dislocation Flows, Irregular Crack Propagation, Locked Crack, Artificial Intelligence, Uphill Climbing Technique. 


\section{BACKGROUND}

Linear fracture mechanics assumes homogeneous microstructure and defect-free manufactured surfaces, which are two idealized material aspects ensuring isotropic mechanical properties. Successive loading of such theoretical models claims that standard crack tip is the most likely to open along its major axis. Accordingly, both the propagation path and the fractured surface are predicted to have smooth features [1-3].

Practical examinations, however, reveal that randomly distributed hard additives, metallic inclusions and impurities are commonly found in the soft matrix of material microstructures. In addition, inspection of finished mechanical products proves that engineering materials are full of core active defects, such as dislocations centers, and of surface active defects, such as sharp cracks. These cracks are of the same order of size as the microstructure--typically less than 10 times grain diameters. Accordingly, material homogeneity can no longer be assumed [4-7].

Ordinary loading of machine parts energizes such types of active defects. External work done unlocks the dislocation centers and induces significant stresses at crack tips. At some load levels, micro displacements of such defects are recorded. The behavior of each individual defect depends on the local microstructure $[8,9]$. More often than not, an advancing defect is bound to face obstacles that steer its direction of propagation. Or the advancing defect may be encircled by a closed set of obstacles that impede any further advance. In such fields, it is hard to theoretically predict the behavior of the moving defect as to whether it may advance or lock, and thereby isotropic properties can no longer be adopted either [10].

Since the path of a moving defect is somehow correlated to the measured roughness of the final fractured surface, a useful visualization of the defects' paths is achieved. Real measurements of fractured surfaces with a noticeable rough appearance prove that these defects often follow an uneven irregular path. Accordingly, examination and analysis of fractured surfaces become a dependable methodology of successful research attempts to pinpoint the cause and mechanism of a fractured product. However, recent models to interpret the behavior of a locked defect, whether a dislocation center or a crack tip, still face some challenges [11,12].

In this study, a concept is so adopted that there is always a pre-determined variable potential line at the early moment before a micro defect shifts its current position. In practice, this is simply the line of the highest average stress. Defects advance so as to reduce the area of the highest average stress. This is a path where the minimum strain energy is consumed to reinstate the equilibrium of material structure. In our model, we prescribed this line beforehand as the line connecting two distant cracks across surface boundaries of a loaded specimen. In the presence of two moving cracks, each crack forms a potential field for the other. The potential field of one crack governs the other crack so that the two gradually approach one the other. Thus, the two cracks have the same objective, which is to reduce the area of the highest average stress. The objective turns out to be an artificial intelligence goal in which two moving competitors try to eventually meet somewhere. In addition, in a medium full of random constraints (obstacles), this objective is still constrained by searching the optimum path. Before one defect changes its position, the magnitude and direction of its local micro displacement should also be locally optimized. Local optimization is based on neighboring obstacles, local stress field, and strain hardening history. These local constraints cause temporary deviations from the governing potential line. Since the paths of the two defects are irregular, the 
governing potential line successively varies in magnitude and direction. For instance, in the case that both defects finally encounter, their common potential line vanishes.

\section{PAST-FRACTURE MECHANISMS}

\subsection{Dislocation Gliding}

During solidification of melted alloys, metallic inclusions and additives freely move in the space of the soft matrix. At numerous locations and simultaneously, crystals and grains nucleation starts with their well organized structures. Different orientations of the resulting crystals lead to random intersection at their boundaries. Grain boundaries are thus full of locked dislocations with stored strain energy. For a thick plate subject to different levels of uniform tensile loading below failure values, external work done helps to gradually unlock such dislocations and to dissipate their strain energy as strain waves. For instance, plane strain state is the basis of the explanation of plastic deformation in metal crystals. Thus if mechanical means are imposed to open a gap of size $\delta$ between two dislocated faces of a ring crystal, the ring becomes in a self-strained state called an edge dislocation. In material science, $\mathrm{W}$ is the total strain energy of the crystal per unit axial length, commonly expressed as [13]

$\mathrm{W}=\left[\mathrm{E} \delta^{2}\right] \mathrm{h} /\left[8 \pi\left(1-v^{2}\right)\right]$

where

$h=\log (R / r)-\left(R^{2}-r^{2}\right)\left(R^{2}+r^{2}\right)^{-1}$,

$\mathrm{E}$ is Young's modulus, $v$ is Poisson's ratio, $\mathrm{R}$ is the ring outer radius related to the overall dimensions of the crystal, and $r$ is the ring inner radius related to the atomic spacing in the crystal lattice. It is established that a dislocation is best displaced with $\delta$ along the path requiring the lowest $\mathrm{W}$. If the loaded plate specimen includes two nearby dislocations with different signs, the line joining their centers acts as a potential field enhancing their mutual attraction, and thereby it represents the most likely theoretical path for dislocations to flow and to release their strain energy. However, since the dislocation will turn around random obstacles, the flow path is commonly irregular.

\subsection{Propagation of Surface Cracks}

Most manufacturing processes induce micro cracks with different sizes to external surfaces of machine parts. For a thick plate subject to tensile loadings below failure levels, stress concentration factors $\gamma$ near crack tips may cause considerable stresses close to plastic yielding. Fig.(1) illustrates that depending on the location $\beta$ and orientation $\theta$ of the crack axis, surrounding cells will have different values of $\gamma$. At the crack external elliptical surfaces with $a, b$ major and minor axes, respectively, the radial stress is null. However, the tangential (opening) stress $\sigma_{t}$ for all points lying on the perimeter of the elliptical surfaces governs the $\gamma$ to be [13] 


$$
\begin{aligned}
& \gamma(\theta, \beta)=\sigma_{\mathrm{t}} / \mathrm{S}=\mathrm{Q}\left[\operatorname{Sinh} 2 \alpha-\left(\mathrm{e}^{2 \alpha} \cos 2 \beta\right.\right. \\
&\left.-1) \cos 2 \theta+\mathrm{e}^{2 \alpha} \sin 2 \beta \sin 2 \theta\right]
\end{aligned}
$$

where

$$
Q=\cosh 2 \alpha-\cos 2 \beta]^{-1} \text {, }
$$

The crack geometrical parameter $\alpha$ is found from tanh $\alpha=b / a$, and $S$ is the average tensile stress expressing a global potential near crack tip. Figs. $(2,3)$ illustrate some relative values of $\gamma$. The figures are intended to prove that for a crack on the left edge of a tensioned plate, the direction of a new open crack is to the right. If there are no obstacles facing the crack tip, crack extends along its major axis. If there are obstacles facing the tip, another new crack tip is opened in the right surface of the active crack. In both cases, at a certain external critical load $S_{c r}$, the active crack should open and start propagation intermittently or continuously towards plate fracture as two separate parts. The work done by external loadings is converted to a necessary surface energy of the extended crack length. In the presence of two surface cracks, the line joining their tips has the highest average stress distribution, and thereby it represents the most likely theoretical path for cracks to propagate along. As in section 2.1 we refer to this line as a potential field governing the dual motion of the crack tips.

\section{AN ARTIFICIAL INTELLIGENCE FRACTURE MODEL}

\subsection{Design of the Model}

Fig.(4) shows a model for a thick plate whose plane area is divided into a matrix of squared cells. Two cells $L$ and $R$ at the side boundaries are arbitrarily selected to simulate the two surface cracks. Based on a uniform probability distribution with a selected mean density, some cells are cleared for dislocations or cracks to pass by, whereas the others are impaired to be traversed. As the plate is loaded in tension, cells along the line potential field L-R have the highest average stress, and cells surrounding crack tips have high stress concentration factors $\gamma$ based on eqns.(1-4) and Figs. $(2,3)$. Thus, the local displacement of a given dislocation center or a crack tip from a current cell to a next adjacent cell depends globally on the current line of the potential field and locally on individual values of strain energy or $\gamma$ at surrounding cells. The dependence is mainly to minimize the strain energy of each minor displacement to achieve a final engagement of the two moving points (fracture).

Based on Artificial Intelligence topics, a similarity may be formulated between a method, called Uphill Climbing, and micro displacements of dislocations and cracks. In the stated method, two competitors (two defects) move in a matrix randomly filled with obstacles. They aim to meet based on sticking to the shortest updated path.

\subsection{Assumptions and Model Limitations}

Without a loss of generality, four assumptions will be included in the proposed model. First; due to the rectangular shape of the cells, a surface crack, e.g. at the L-edge, is surrounded by only five cells. Thus it can propagate along only five directions, being 
$\theta=\pi / 2, \pi / 4,0,-\pi / 4,-\pi / 2$. This corresponds to only seven cells for a core crack. Second, any new path can safely cross a previous path at $45^{\circ}$. Third; path deviations are preferred towards the current potential line. Fourth, in the case that the two cracks tips lie in one row, the preferred advance occurs along their common row. While a crack follows this row path and accidentally confronts an obstacle forcing it to deviate at $45^{\circ}$, the best selected cell is the closer one to the original potential line.

\subsection{Running of the Model}

The obstacles are first randomly distributed over the matrix. The type of the random law and the density mean value are both arbitrarily selected. The law of randomness may be Gaussian, Raleigh, or uniform, being the one selected in the next case studies. The mean density level is expressed as the percentage of impaired cells in a $25 X 18$ cellular matrix or as the number of obstacles per unit plate area. For instance, $20 \%$ obstacles or equivalently a density level of 2 means that we may find 2 obstacles in every 10 adjacent cells on the average. A defect is incapable or is not active enough to cross these cells. In real microstructure, these impaired cells may model hard inclusions, or spots with high compressed stresses, or complex fields of involved dislocations and grain boundaries.

In order to run the model, one of the two cracks is activated via two steps. For instance, the first step is a global estimation in which the left crack, or left-dislocation center, globally estimates its location in the potential field of the right crack. This estimation results in reinstating an updated direction towards its new ultimate destination, remaining distance to meet, direction, and speed. The second step is a local estimation to assess the best micro displacement or neighbor cell to advance in order to follow the results of global estimation. The second step is expressed in terms of Artificial Intelligence language as

Cell left $(i, j)$ new $=$ min potential cell left $(i, j)$ old w.r.t cell right (new)

where

$-1 \leq \mathrm{i} \leq 1$, and $-1 \leq \mathrm{j} \leq 1$

Subject to $i \neq$ ja nd cell left (ij) new is clear,

meaning that cell left (i,j) new should have no original obstacles and it should not be previously traversed by another defect. If there is not a cell satisfying the conditions of eqn.(5), the left crack (or left-dislocation center) is locked, and the two traveled path segments do not join for the applied load level. If there is, the left crack tip eventually occupies a new cell, marking one cell unit extension.

The right crack (or the right-dislocation center), in turn, is allowed to carry out two identical steps in order to extend just one cell based on the same criterion in eqn.(5). Notice that the right crack aims at the last cell recently occupied by the left crack. This means that the relative objective of one crack is variable and depends on the best move each time the other crack selects. Successive moves are carried out by only one crack at a time.

The contest ends up with one of two states. First, both cracks succeed to achieve their goals as they meet at one common core cell Second, both cracks fails to meet when one crack is entrapped or encircled by the originally distributed obstacles or by 
the strained cells previously crossed by a dislocation flow and dislocation energy release. A disabled or a captive crack is a special case which arises when one crack is locked at its original surface spot owing toencirclement by impassable cells.

\section{APPLICATIONS OF THE MODEL}

Figs.(5) shows the matrix with a uniform random distribution of obstacles with a mean density $20 \%$. As we notice, the number of impassable obstacles or impenetrable cells, marked as blackened, is small and they are sparsely distributed over the whole $25 \times 18$ cellular matrix. The two surface cracks are arbitrarily selected on the vertical side boundaries, being marked at cells by "L" for left and "R" for the right one. Just to simplify the analysis, both cracked cells are selected to initially lie along the same row. The geometry of both cracks is considered of the elliptical type with the tip pointing horizontally. The plate is uniformly loaded by tensile stresses at the upper and lower edges. The conditions of $100 \%$ full loading refer to crack continuous propagation towards impending fracture of the plate into two separate parts. Application of a percent of the full load is assumed to only cause dislocation preliminary settlement flow. Three preliminary load levels are chosen for illustration; namely $25 \%, 50 \%$, and $75 \%$ of the fracture full loading.

The favored direction of propagation is the line of the highest average stress, being the line L-R. Since linear elasticity assumes homogeneous structure and isotropic properties, this straight line is the ideal target path of the moving dislocations or cracks, and thereby is predicted to be the future fractured even surface as well (no irregularities).

\section{ANALYSIS OF RESULTS}

Fig.(6) shows the paths of strained cells under the four levels of loading for $20 \%$ obstacles density. For the $25 \%$ load, the figure shows the paths of the two edge dislocations flows starting from surface boundaries towards the plate core. The two paths pass through the clear cells and turn around impaired cells. These already traversed cells on the path undergo strain hardening and will resist any future crossing attempts. In this preliminary case the two paths meet near the plate symmetric axis, marked by a blackened symbol. Under the $50 \%$ load level, a second flow of strain hardening of dislocation is now constrained by the original obstacles besides the first strained path. As a result, a new $50 \%$ path is shown in Fig.(6). At a $75 \%$ load level, dislocation motion is more constrained to avoid original obstacles, $25 \%$ and $50 \%$ strained cells as well. Fig.(6) illustrates the $75 \%$-load dislocation settlement path. Upon applying the $100 \%$ full load, the stress intensity at the crack tip reaches the fracture toughness and both cracks propagate based on the opening mode $(\mathrm{I})$, leading to a rupture path of the plate into two separate parts.

\section{DISCUSSION}

Low levels of loading provide strain energy necessary to unlock dislocations in the neighbor cells of the two cracks, leading to a path of strain hardened cells. It is evident that microstructure dislocations flow from surface cells to intersect one 
another at core cells For $20 \%$ obstacle density, Fig.(6) shows that any left-hand dislocation finishes its flow at the symmetric axis of the loaded plate, where it meets the end-flow of the counterpart right-hand dislocation. This means that the resistance against dislocation flow is direction-independent. However, for the three dislocation pairs of the $30 \%$ obstacle density, Figs. $(7,8)$, any two path segments are unequal which reflects direction-dependent resistance.

As far as $100 \%$ fracture load is concerned, Fig.(6) for $20 \%$ obstacle density shows that the pair of propagating crack tips are early locked at far distant locations. Since the two path segments are not connected, extra loading will cause sudden separation over the line joining the last two positions where the crack tips are locked. Accordingly, the fracture surface will have two areas with different texture; the first belonging to slow crack advancing and the second belonging to a final sudden separation. Nevertheless, Fig.(8) for $30 \%$ obstacle density illustrates that the pair of propagating crack tips do meet, which means that the fracture surface belongs wholly to a slow mechanism of crack advancing.

\section{CONCLUSION}

Using Cartesian coordinates for square cells is adopted for simple formulation, computer speed, and memory limitations. This approach causes a limited number of chosen directions for a crack to follow, being only 7 directions for a core crack and 5 directions for a surface crack. Therefore, the resulting path has a rough accuracy and only serves illustrative purposes. If cells are shaped and located using polar or elliptical coordinates, more directions are allowed for a crack to follow, and loading may be applied with infinitesimal increments. Such a suggested refined model would yield a more accurate detailed path. If the two cracks tips are to meet, propagation proceeds gradually based on optimization of local and global factors. If the hardening mechanism is active it will enhance crack entrapping or locking. As density of obstacles increases, i.e. the structure hard inclusions become dense in the soft matrix, the cracks hardly propagate towards meeting, and thereby they are likely to be entrapped.

The model can be further refined in two forms. First; the model starts by randomizing the obstacles in the cellular matrix. After each crack displaces, a modified obstacle shift is introduced from itslast position as to include a dynamic feature of the obstacles. The obstacles' motion may be directional, to adapt their positions based on the crack motion, or may be random.

Second; the obstacles are distributed based on a given random law in the matrix, here the uniform random distribution is selected. The structure is then assumed homogeneous on the global level but heterogeneous on the micro level. However, the obstacles distribution can be made to be biased or clustered globally over certain areas. Under such not-ideal and abnormal conditions resulting from imperfect metallurgical or mechanical processes, the model presents a good method to observe how cracks may behave. For instance, one of the two cracks may exist in an area with higher obstacle density than the other crack. In the presence of more than two surface cracks, evaluation of their different potential lines determines which cracks are likely to propagate and which cracks are likely to meet. By implementing the model with several types of random distribution laws, it is possible to eventually correlate the hard-to-measure random distribution of hard inclusions or clustered dislocations to the easy-to-measure roughness of fractured surfaces. 


\section{REFERENCES}

[1] K. Kaddouri, M. Belhouari, B. Bachir Bouiadjra and B. Serier, "Finite element analysis of crack perpendicular to bi-material interface: Case of couple ceramicmetal." Computational Materials Science, Volume 35, Issue 1, pp 53-60, January 2006.

[2] Vladislav Levkovitch, Rainer Sievert and Bob Svendsen, "Application of extended crystal plasticity to the modeling of glide and kink bands and of crack opening in single crystals. "Computational Materials Science, Volume 32, Issues 3-4, pp 426434, March 2005.

[3] David Taylor, "Modeling of fatigue crack growth at the microstructural level." Computational Materials Science, Volume 25, Issues 1-2, pp 228-236. September 2002.

[4] E. Ducourthial, E. Bouchaud and J. -L. Chaboche, Influence of microcracks on a propagation of macrocracks, Computational Materials Science, Volume 19, Issues 1-4, pp 229-234, December 2000.

[5] S. Hönle and S. Schmauder, "Micromechanical simulation of crack growth in WC/Co using embedded unit cells," Computational Materials Science, Volume 13, Issues 1-3, pp 56-60, December 1998.

[6] Priya Vashishta, Aiichiro Nakano, Rajiv K. Kalia and Ingvar Ebbsjö, "Crack propagation and fracture in ceramic films-million atom molecular dynamics simulations on parallel computers," Materials Science and Engineering B, Volume 37, Issues 1-3, pp 56-71, February 1996.

[7] G. Prokopski and B. Langier, "Effect of water/cement ratio and silica fume addition on the fracture toughness and morphology of fractured surfaces of gravel concretes," Cement and Concrete Research, Volume 30, Issue 9, pp 1427-1433, September 2000.

[8] J. H. Kim and S. B. Lee, "Behavior of plasticity-induced crack closure and roughness-induced crack closure in aluminum alloy," International Journal of Fatigue, Supplement, Volume 23, 1, pp 247-251, 2001.

[9] G. Papakaliatakis, "Computational study of the initiation of crack extension in a solid propellant with a circular hole or inclusion," Mathematical and Computer Modeling, Volume 42, Issues 7-8, pp 727-738, October 2005.

[10] V. Tvergaard and A. Needleman, "Three dimensional microstructural effects on plane strain ductile crack growth, "International Journal of Solids and Structures, In Press, Corrected Proof, Available online 24 August 2005.

[11] Jun Lei, Yue-Sheng Wang and Dietmar Gross, "Analysis of dynamic interaction between an inclusion and a nearby moving crack by BEM," Engineering Analysis with Boundary Elements, Volume 29, Issue 8, pp 802-813, August 2005.

[12] H. Tang, A. Acharya and S. Saigal, "Directional dependence of crack growth along the interface of a bicrystal with symmetric tilt boundary in the presence of gradient effects," Mechanics of Materials, Volume 37, Issue 5, pp 593-606, May 2005.

[13] Timoshenko, S.P., and Goodier, J.N., "Theory of Elasticity," McGraw-Hill Inc., Third Edition, 1970. 


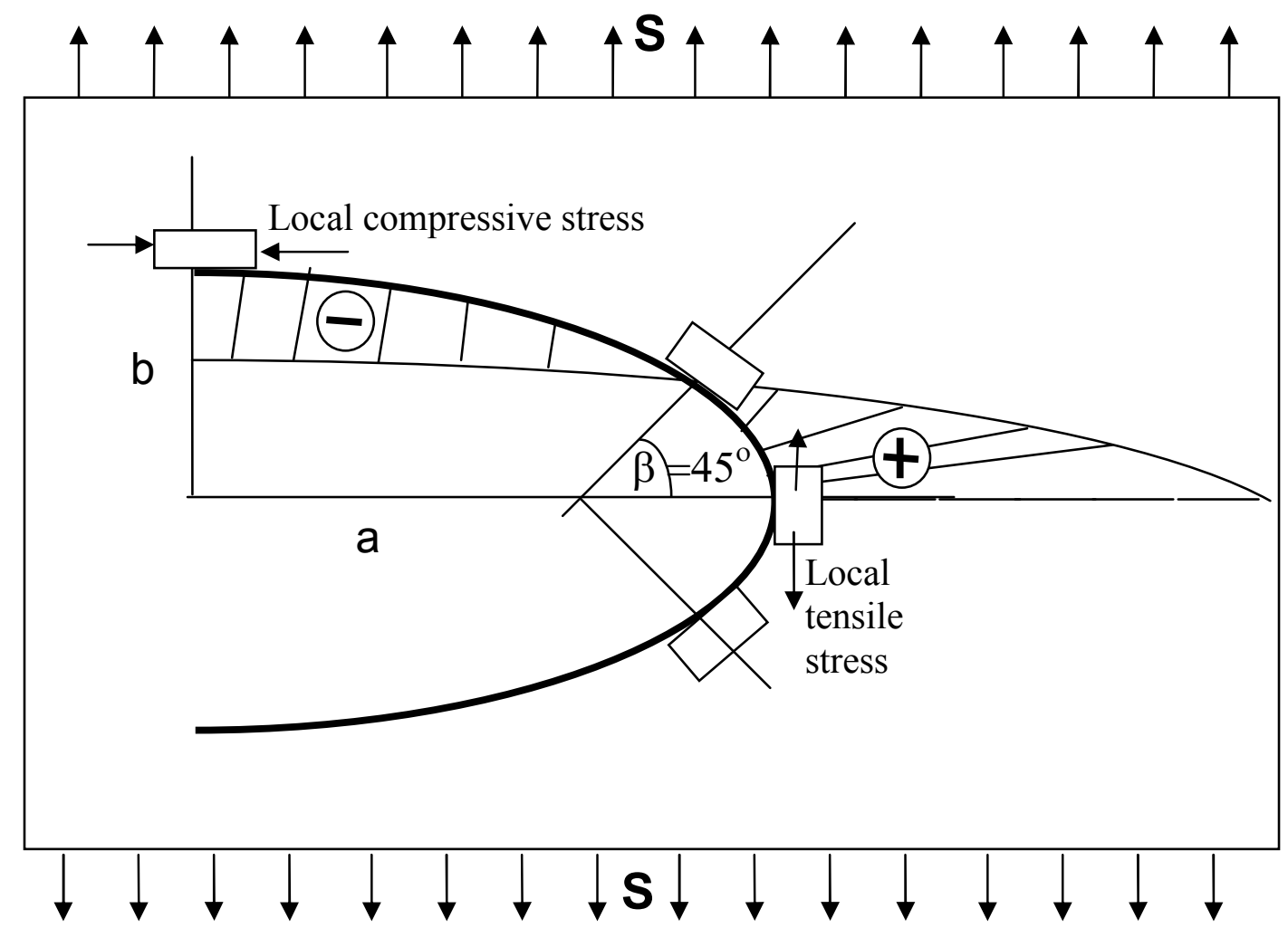

Fig.1 Profile of stress distribution along the surface of an elliptical crack

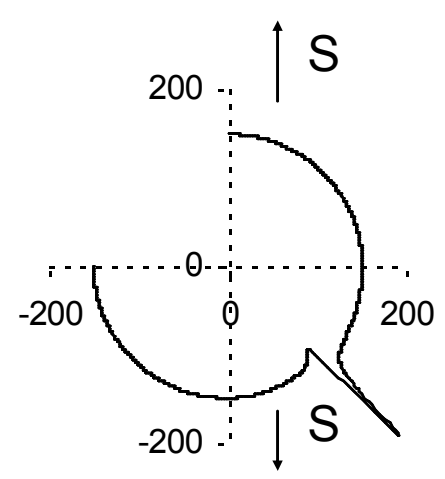

a) South-east propagation

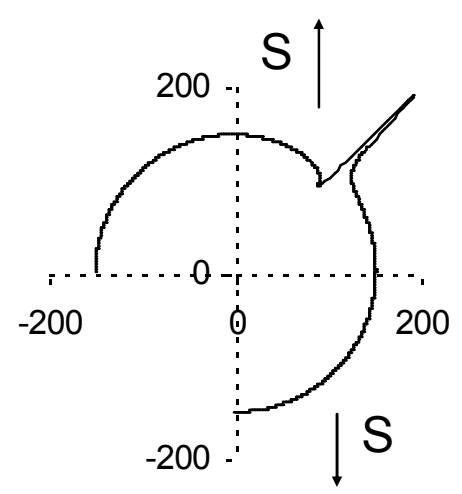

(b) North-east propagation 


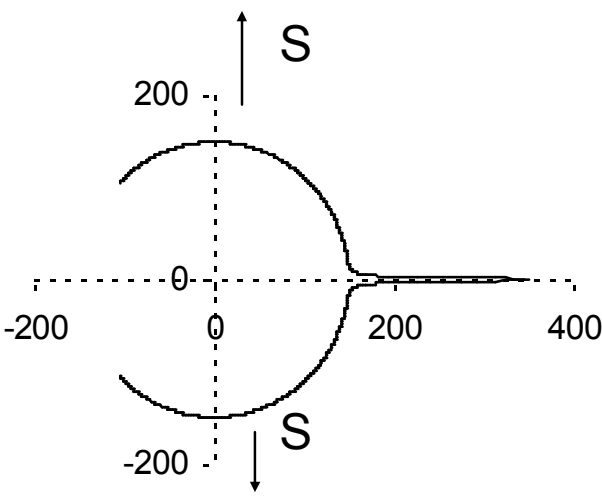

(c) Eastern propagation

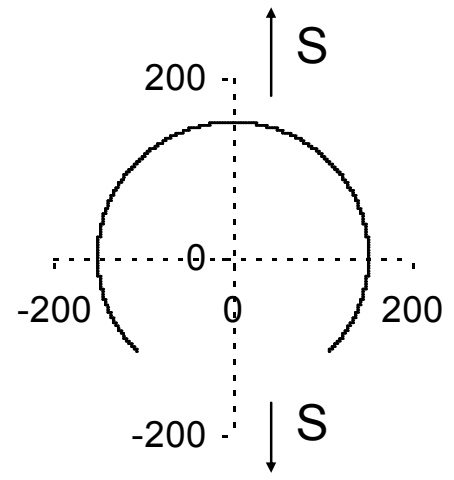

(d) Northern propagation

Fig.2 Stress concentration $\gamma$ near the tip of an elliptical crack, relative illustration based on eqn.(3) 


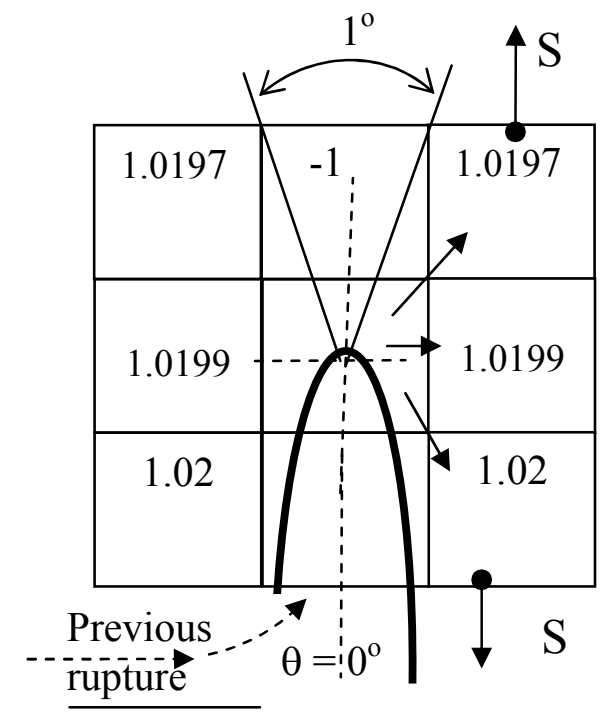

(a) tip pointing to north

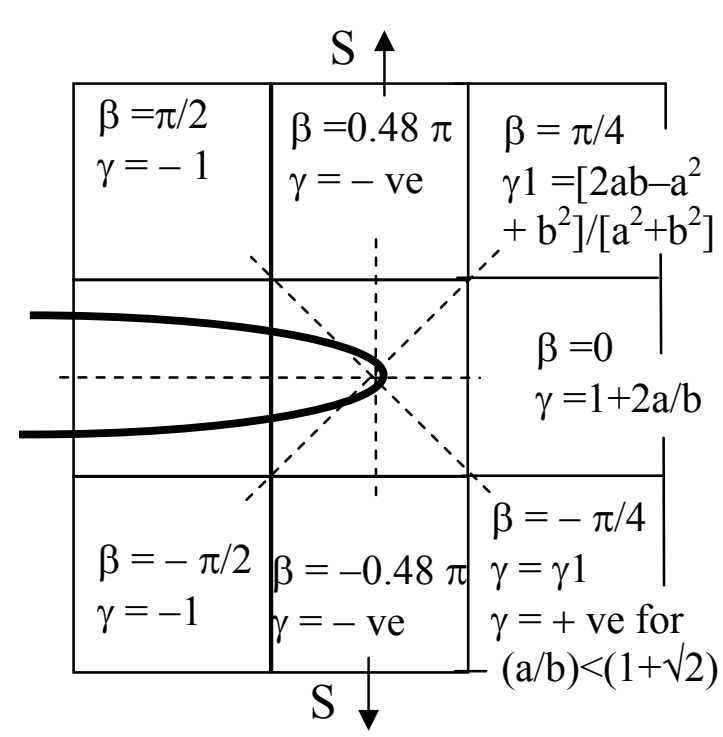

(c) tip pointing to east, $a$ \& $b$ from Fig.(1)

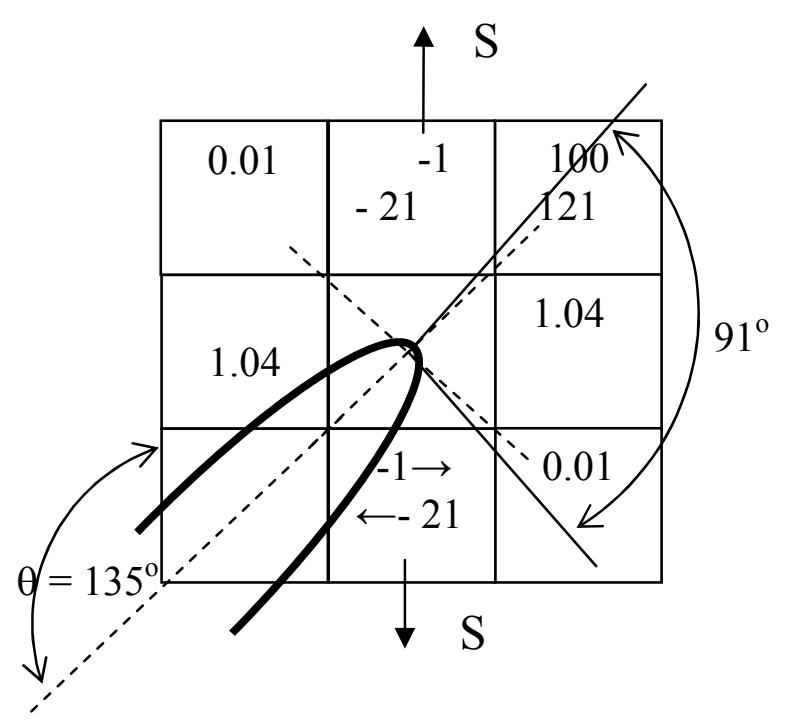

(b) tip pointing to north-east

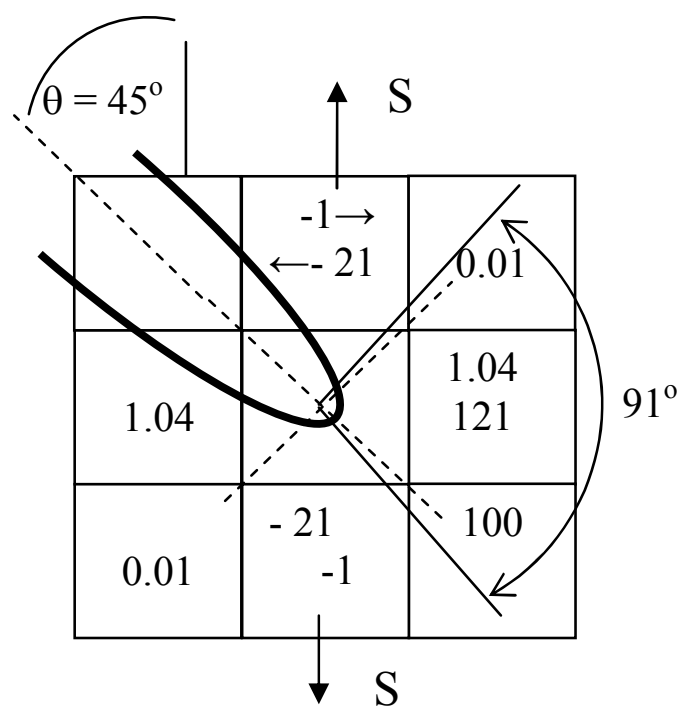

(d) tip pointing to south-east

Fig. 3 Numerical values of stress concentration factor $\gamma$ at the 7 cells surrounding a modeled crack tip, based on eqn.(3) 
S

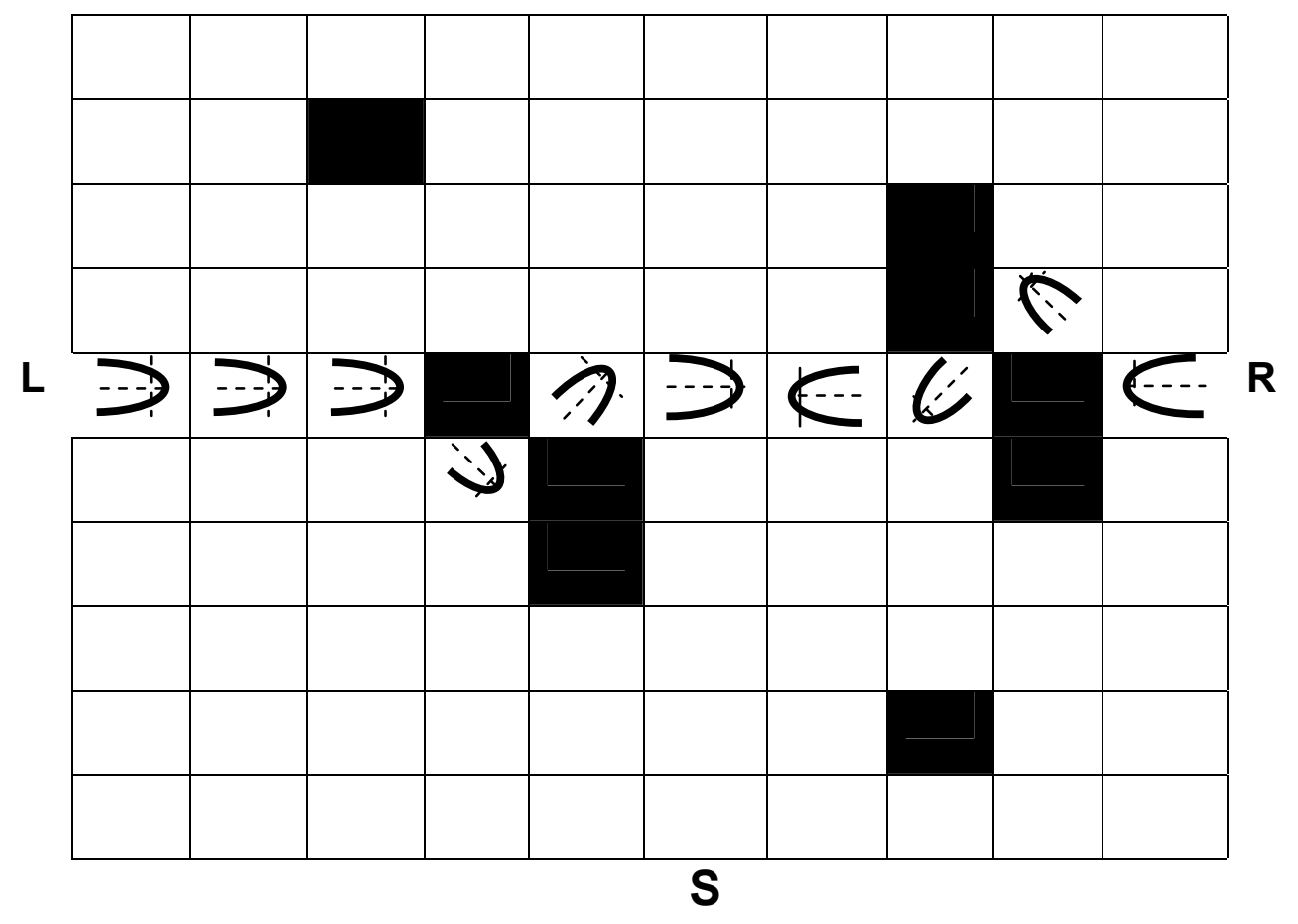

Fig.4 successive directional changes of two cracks' axes to propagate while avoiding resisting obstacles, an artificial intelligence approach

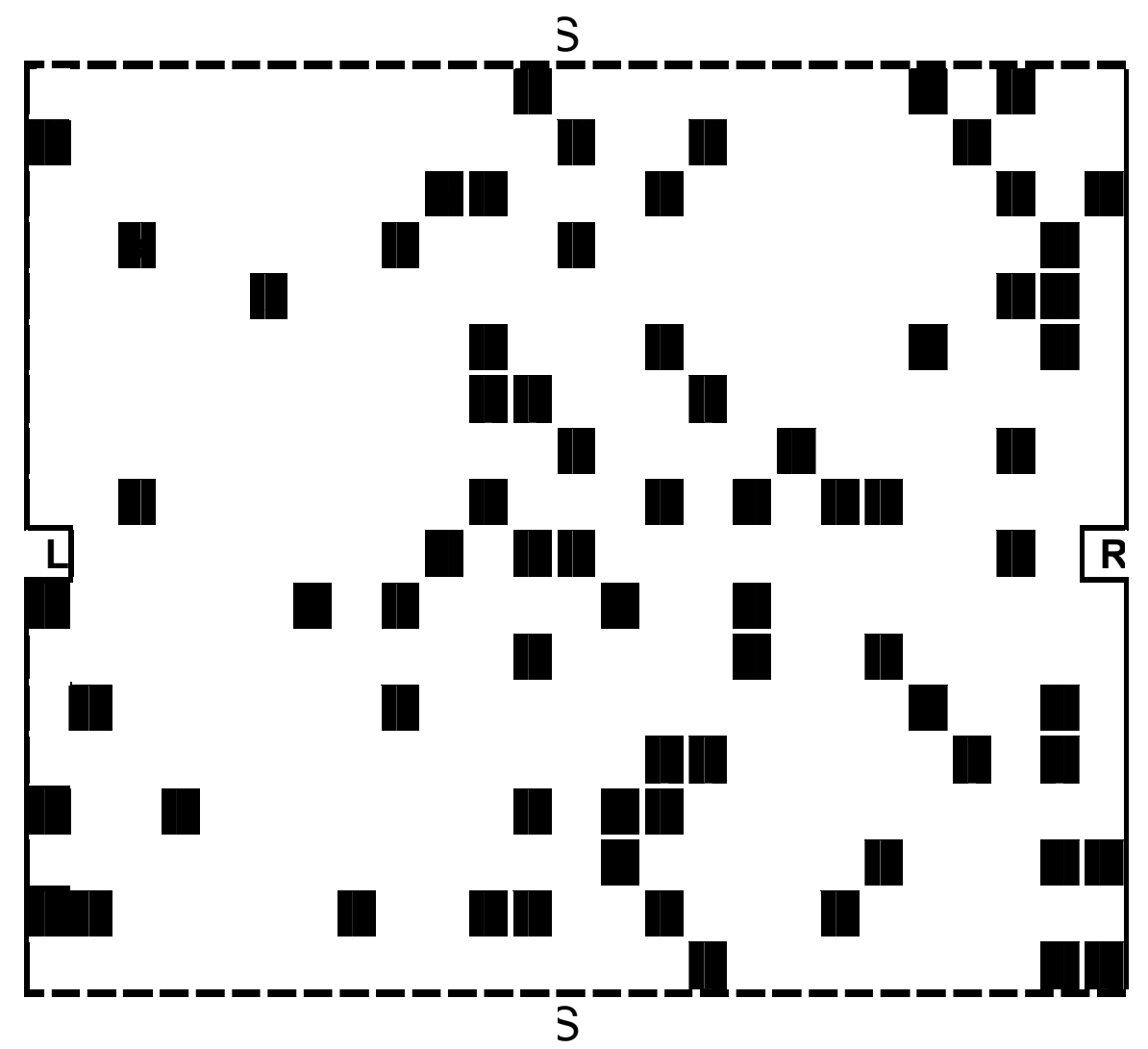

Fig.5 A 25x18 Cellular Matrix With 20\% Randomly Impaired Cell 


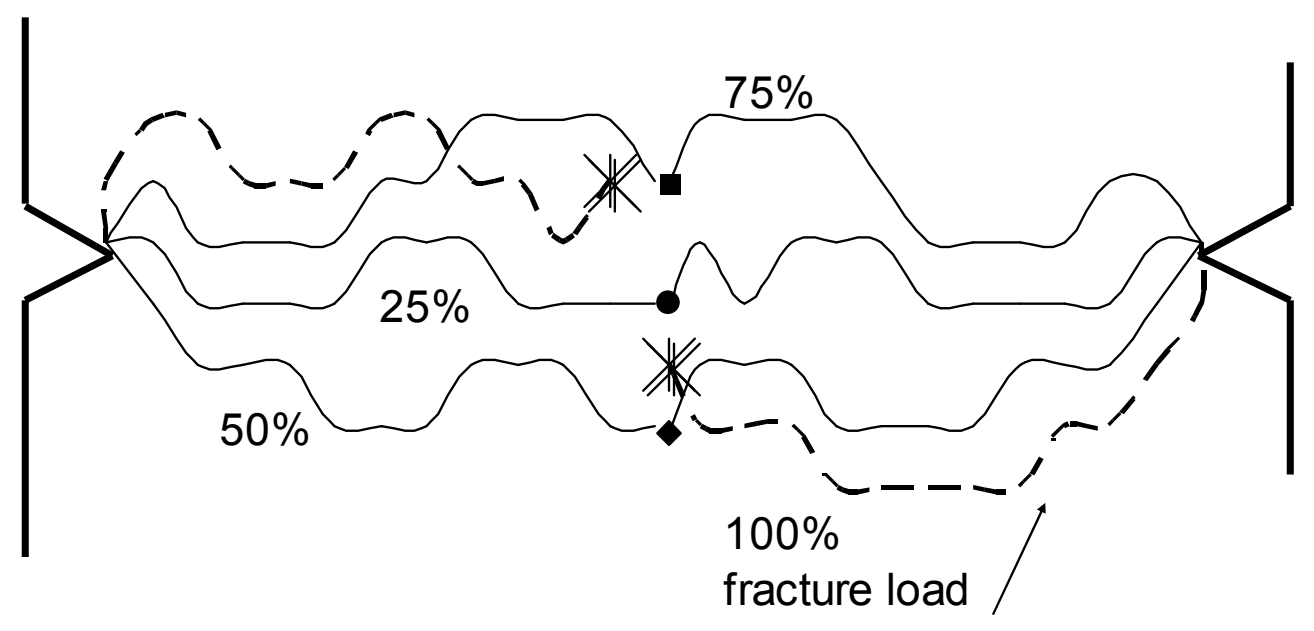

$\begin{array}{llllll}0 & 5 & 10 & 15 & 20 & 25\end{array}$

Fig.(6) Dislocation flows and crack propagation for $20 \%$ obstacles density

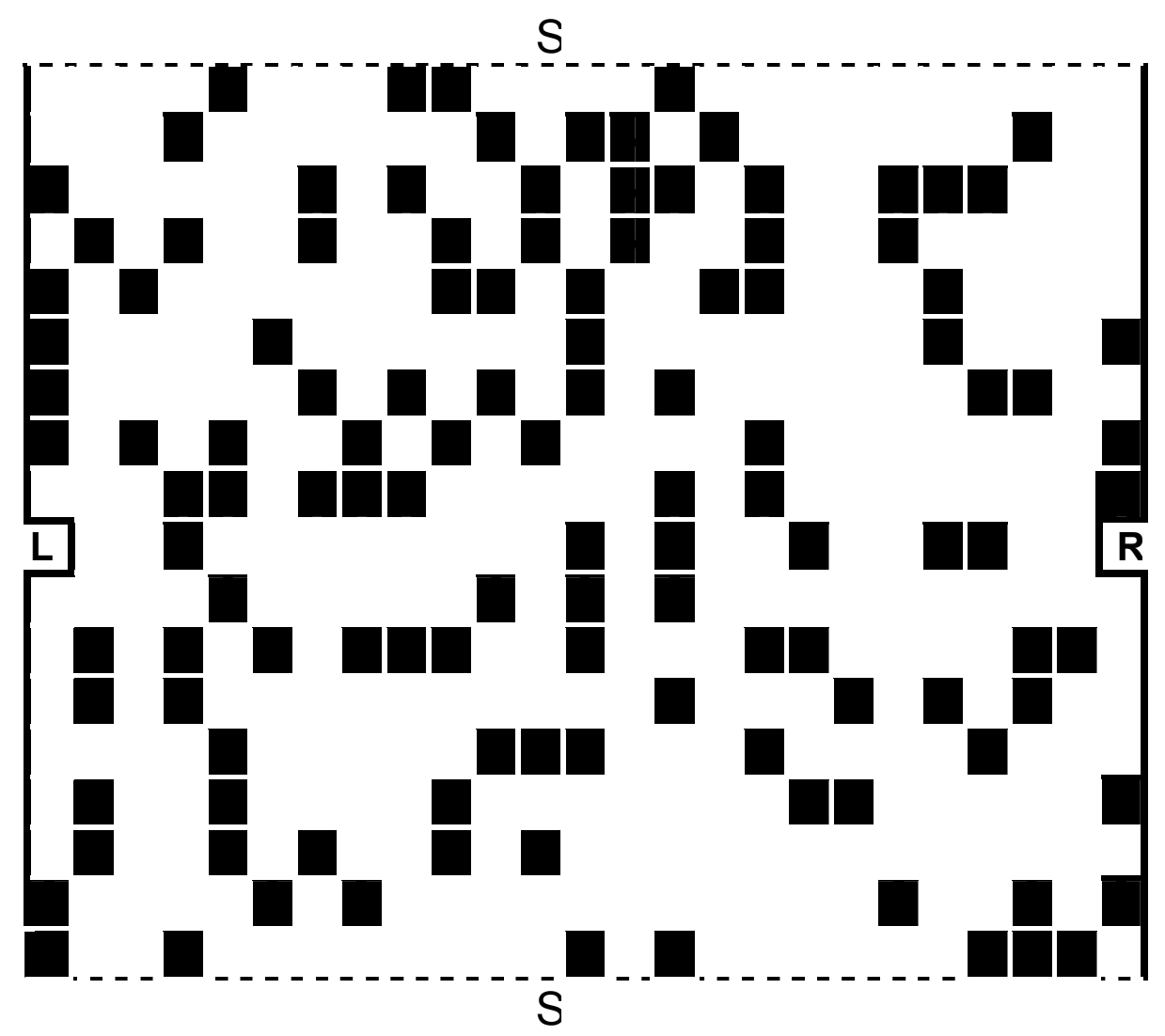

fig. 7 a $25 \times 18$ cellular matrix with $30 \%$ randomly impaired cells 


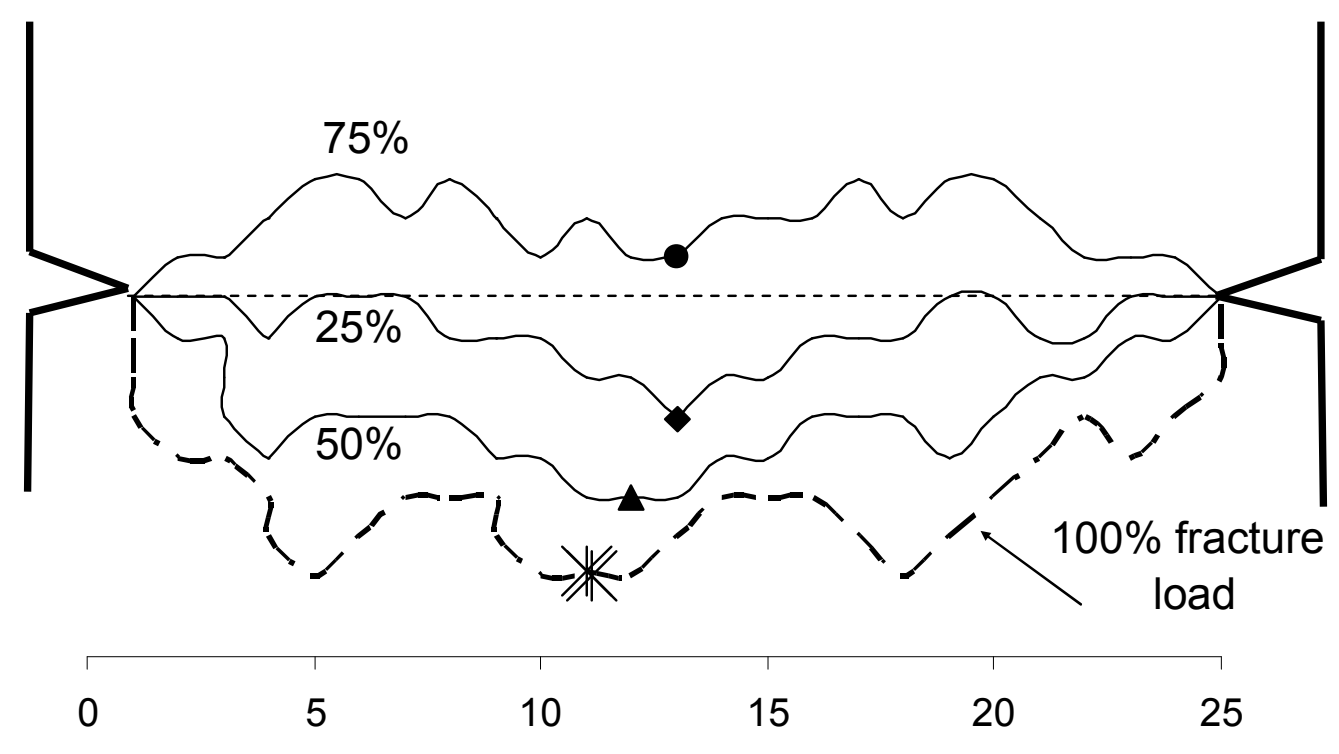

Fig.(8) Dislocation flows and crack propagation for $30 \%$ obstacles density 